BOOK REVIEW

\title{
CALCULATIONS IN LABORATORY MEDICINE
}

\section{Author: Allan Deacon, Edited by Roy Sherwood and James Hooper. ACB Venture Publications 2009. 338 pp. ISBN 978-0-902429-43-7. Price $£ 35.00$. Available from the Association of Clinical Biochemists, 1300-132 Tooley St., London SE1 2TU}

Reviewer: Joseph Lopez

This is yet another title from the impressive series on topics in clinical biochemistry that is published by the ACB. The author is a senior clinical biochemist who was motivated to write this book after seeing the poor numeracy skills of trainees in laboratory medicine. It covers a wide range of areas and starts with the very basics, i.e. units. The first chapter opens with the aphorism, "a numerical result without units is meaningless" and then proceeds to explain the SI units for the dimensions of length, mass and time and the conversion of mass units to SI units. The next chapter deals with another fundamental area, that of preparation of solutions and dilutions.

Subsequent chapters are on acid-base, $\mathrm{pH}$ and buffers, spectrophotometry, renal function and osmolality. The chapter on enzymology covers the calculations related to classical enzyme kinetics, the Michaelis-Menten equation, inhibition and the calculation of enzyme activity. There is a chapter on basic pharmacokinetics. Though not be an area that most clinical biochemists would deal with, it is nonetheless helpful background for those who may work in therapeutic drug monitoring. Clinicians will find this chapter useful and that on body fluids and electrolytes.

There are five chapters on statistics on medical statistics covering the analysis of means and variance, correlation and regression, the clinical utility of laboratory tests and statistical power. They take up approximately one-third of the book. Though these topics may be found in most books on medical statistics, the examples in these chapters are drawn from clinical biochemsitry.

\section{Address for Correspondence :}

\section{Mr. Joseph Lopez}

Head, Department of Biomedical Sciences

MAHSA University College

Kuala Lumpur, Malaysia

E-mail : jblopez@streamyx.com
The last chapter gives examples of a variety of miscellaneous calculations: the section on recovery experiments leads this chapter and brought back some old memories. One hopes that these are still performed in method validation. I was hard pressed to imagine how often the elimination of a tumour marker is calculated by the average clinical biochemist in

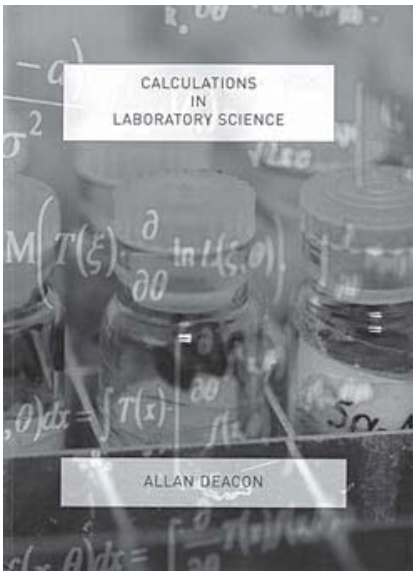
the laboratory though a clinician may have some use for this. Though most diagnostic laboratories have stopped using radioactive isotopes, the calculation of radioactive decay may still be useful for the clinical biochemist who is engaged in some areas of research. Calculations of urinary nitrogen excretion, titrable gastric acidity and internal standardisation in chromatography are briefly mentioned in the chapter. Curiously, the author has included the Hardy-Weinberg equilibrium in population genetics, but I wonder how often a laboratory is likely to need this.

An attractive feature of this book is the numerous examples of calculations that have been worked out in detail after section within each chapter. In addition, there are additional exercises provided at the end of each chapter with answers at the back of the book.

Though readers may be familiar with most of the topics described, they would most likely have learnt it from a variety of sources. The author has done us an important service by collating all of these into a single text for easy reference. This book is clearly written in a manner that should be easily understood by students of all stripes, be they trainee pathologists, scientists, undergraduates or technologists. It is also a very useful reference that every laboratory and medical library should have. 